\title{
Coffee polyphenols exert hypocholesterolemic effects in zebrafish fed a high-cholesterol diet
}

\author{
Shinichi Meguro*, Takahiro Hasumura and Tadashi Hase
}

\begin{abstract}
Background: Hypercholesterolemia is an important risk factor for the development of coronary artery disease. Some dietary polyphenols, such as coffee polyphenols (CPPs), reduce cholesterol levels. The mechanism of this cholesterol-lowering effect is not fully understood, although 5-CQA, a major component of CPPs, reportedly inhibits cholesterol biosynthesis. Here, we investigated the mechanism of the cholesterol-lowering effect of CPPs on the basis of cholesterol metabolism-related gene expression in the liver. We also examined the effects of CPPs on vascular lipid accumulation in zebrafish with high cholesterol diet-induced hypercholesterolemia.
\end{abstract}

Methods: Over 14 weeks, adult zebrafish were fed a control diet, a high-cholesterol diet, or the latter diet supplemented with CPPs. To measure the extent of vascular lipid accumulation, for 10 days larval zebrafish (which are optically transparent) were fed these same diets with the addition of a fluorescent cholesteryl ester.

Results: In adult zebrafish, addition of CPPs to a high-cholesterol diet significantly suppressed the increase in plasma and liver cholesterol levels seen when fish ingested the same diet lacking CPPs. Transcription levels of the liver genes hmgcra (encoding 3-hydroxy-3-methylglutaryl-coenzyme A reductase A, a rate-limiting enzyme in cholesterol biosynthesis) and mtp (encoding microsomal triglyceride transfer protein, a lipid transfer protein required for assembly and secretion of lipoproteins) were significantly lower in fish fed the CPP-containing diet than in fish fed the unsupplemented high-cholesterol diet. In contrast, the expression level of the liver gene cyp7a1a (encoding the cytochrome P450 polypeptide 1a of subfamily A of family 7, a rate-limiting enzyme for bile acid biosynthesis) increased significantly upon consumption of the CPP-containing diet. In larval fish, accumulation of fluorescently labeled cholesterol in the caudal artery was greatly reduced on the CPP-containing diet.

Conclusions: CPP ingestion suppressed cholesterol accumulation in the plasma, liver, and vascular system of zebrafish. Downregulation of cholesterol and lipoprotein synthesis and upregulation of bile acid synthesis in the liver may be the fundamental underlying mechanisms by which CPPs exert their hypocholesterolemic effects. CPP intake may help prevent and manage hypercholesterolemia in humans, and further investigations along these lines using a variety of CPP dose rates are warranted.

Keywords: Chlorogenic acids, Cholesterol diet, Coffee polyphenols, Cytochrome P450 7A1, 3-hydroxy-3methylglutaryl-coenzyme A reductase, Hypercholesterolemia, Microsomal triglyceride transfer protein, Vascular lipid accumulation, Zebrafish

\section{Background}

Cardiovascular disease is the leading cause of morbidity and mortality worldwide. Hypercholesterolemia triggers atherosclerosis and is a major risk factor for the development of cardiovascular disease. Several epidemiological studies have clearly shown that increased levels of plasma

\footnotetext{
* Correspondence: meguro.shinichi@kao.co.jp

Biological Science Research, Kao Corporation, 2606 Akabane, Ichikai-machi, Haga-gun, Tochigi 321-3497, Japan
}

\section{() Biomed Central}

(c) 2013 Meguro et al.; licensee BioMed Central Ltd. This is an open access article distributed under the terms of the Creative Commons Attribution License (http://creativecommons.org/licenses/by/2.0), which permits unrestricted use, distribution, and reproduction in any medium, provided the original work is properly cited. cholesterol-particularly low-density lipoprotein (LDL) cholesterol-are associated with the development of cardiovascular disease $[1,2]$. Importantly, hypercholesterolemia is a modifiable risk factor. Lifestyle changes and ingestion of various dietary compounds-such as green tea, plant sterols, and soy proteins-can reduce hypercholesterolemia [3].

Worldwide, coffee is one of the most widely consumed beverages, and it is an extremely rich source of biologically active polyphenols [4]. Coffee polyphenol (CPP) preparations 
are rich in caffeoylquinic acids (CQAs), feruloylquinic acids (FQAs), and dicaffeoylquinic acids (diCQAs). The physiological activities of CPPs include dose-dependent reduction in blood pressure, improvement of endothelial function, and suppression of diet-induced body fat accumulation and postprandial hyperglycemia [5-10]. Recently, the reduction of cholesterol levels by CPPs and 5-CQA (chlorogenic acid) has been reported [9,11-14]. However, little has been reported about the mechanism of action of these substances: it has been reported only that 5-CQA inhibits cholesterol biosynthesis $[11,14]$. None of the possible effects of CPPs on vascular lipid accumulation-an important early step in atherogenesis-has been studied.

Zebrafish (Danio rerio) organs and tissues are similar to those of humans in both structure and function. Consequently, the zebrafish is increasingly being used as a model of human disease because (a) the fish is readily amenable to genetic manipulation; (b) it breeds readily in captivity; and, (c) experimental colonies can be inexpensively maintained [15]. In addition, several studies have found that the zebrafish is an excellent model of vertebrate lipid metabolism $[16,17]$. Stoletov et al. [18] showed that zebrafish fed a high-cholesterol diet developed hypercholesterolemia, exhibited a remarkable capacity to oxidize lipoproteins and accumulated vascular lipids. They concluded that the zebrafish was a suitable model in which to study the pathological events important in early-stage atherogenesis.

Here, we investigated the effects of CPP ingestion by zebrafish rendered hypercholesterolemic by consumption of a high-cholesterol diet. We measured plasma and liver cholesterol levels, the expression levels of liver genes involved in cholesterol metabolism, and the extent of vascular lipid accumulation. We compared data from test and control fish cohorts.

\section{Methods \\ Preparation of CPP}

CPPs were prepared by hot-water extraction from roasted coffee beans, and CPP composition was determined by HPLC analysis of the extract [9]. The total polyphenol content of the CPP preparation was $77.1 \%(\mathrm{w} / \mathrm{w})$. Individual polyphenol compositions were (all w/w): 7.7\% 3-CQA; 15.5\% 4-CQA; 31.9\% 5-CQA; 7.6\% 3- FQA; 5.6\% 4-FQA; 8.5\% 5-FQA; 9.0\% 3,4-diCQA; 8.9\% 3,5-diCQA; and 5.3\% 4,5-diCQA. The CPP powder contained no caffeine.

\section{Animals}

Adult zebrafish were purchased from a local pet supplier (Meito Suien Co., Ltd., Remix, Nagoya, Japan). All fish were raised and maintained on a 14:10-h light:dark cycle at $28^{\circ} \mathrm{C}$, and water quality was maintained as described in The Zebrafish Book [19]. All fish were handled in strict accordance with the Guidelines of the Animal Care
Committee of Kao Corporation, The Zebrafish Book, and the Guide for the Care and Use of Laboratory Animals (8th edition) [20].

\section{Diets}

The control diet contained 75\% (w/w) standard zebrafish chow (Otohime B2; Marubeni Nisshin Feed Co. Ltd., Tokyo, Japan) and 25\% (w/w) gluten (Wako Pure Chemical Industries, Ltd., Osaka, Japan). The high-cholesterol diet was the control diet with the addition of $4 \%(\mathrm{w} / \mathrm{w})$ cholesterol (Wako Pure Chemical Industries). The "CPP diet" was the control diet with the addition of both $4 \%$ $(\mathrm{w} / \mathrm{w})$ cholesterol and $5 \%(\mathrm{w} / \mathrm{w})$ CPPs. To measure the cholesterol content of the Otohime B2 chow, lipids were extracted by using the conventional Folch method [21]. The cholesterol content of the control diet before the addition of cholesterol was $0.4 \%(\mathrm{w} / \mathrm{w})$.

\section{Experimental design}

Experiment 1: Female adult zebrafish 6 months postfertilization were weighed under anesthesia with $0.005 \%$ (w/v) tricaine (Sigma-Aldrich, MO, USA) and allocated to three groups of 7 or 8 fish with similar body weights. Each group was placed in a 1.7-L tank. The three groups were fed the control, the high-cholesterol, or the CPP diet twice daily (20 $\mathrm{mg}$ of food/day per fish) over 14 weeks. During feeding, water inflow to the tanks was paused for $45 \mathrm{~min}$ and the fish were allowed to consume their diet for $30 \mathrm{~min}$. On the last day of the experiment, all fish were euthanized, body weights were measured, and blood samples were taken from each caudal artery (into heparinized glass capillaries) and dissected livers. Samples from each liver were stored in duplicate for analysis of lipid levels and mRNA expression patterns.

Experiment 2: Twice daily for $30 \mathrm{~min}$ for 10 days, zebrafish larvae (collected 5 days post-fertilization) were fed either the control, the high-cholesterol, or the CPP diet supplemented with $0.002 \%(\mathrm{w} / \mathrm{w})$ cholesteryl BODIPY 576/589 C11 (Life Technology, CA, USA). The larvae were maintained in egg water (water containing 0.6 $\mathrm{g} \mathrm{NaCl} / \mathrm{L}$ ) at $28^{\circ} \mathrm{C}$ under light-shielded conditions. The egg water was changed after each feeding. On the final day of the experiment, 10 randomly selected larvae from each group were collected and anesthetized by being placed in a small drop of $0.005 \%(\mathrm{w} / \mathrm{v})$ tricaine; they were then placed onto a glass slide and immediately transferred into the light-shielded chamber of a fluorescence microscope (BZ-9000; Keyence Corporation, Osaka, Japan), and a fluorescence image of the caudal artery $(1000 \mu \mathrm{m}$ caudally from just above the anus) was photographed. The specifications of the BZ-9000 were as follows: light source, ultra-high-pressure mercury lamp (120 W); fluorescence filter, BZ filter TRITC (excitation wavelength $540 \mathrm{~nm}$, absorption wavelength $605 \mathrm{~nm}$ ); objective, Nikon CFI 
60 series $(\times 10)$; and camera, 2/3 inch, 1.5 million pixel monochrome CCD. To ensure sufficient sensitivity of the comparisons of fluorescence among larvae, each larva was placed on the stage for no more than $1 \mathrm{~min}$. The mean fluorescence intensity of the caudal arteries of each group was quantified by specifying the range of fluorescence of the caudal artery with the aid of inbuilt BZ-II Analyzer software (Keyence Corporation).

\section{Analysis of plasma and hepatic lipid levels}

Plasma was obtained by centrifugation of blood samples at $1500 \mathrm{~g}$ for $5 \mathrm{~min}$ at $4^{\circ} \mathrm{C}$. Liver lipids were extracted by using the conventional Folch method [21]. Cholesterol levels in plasma and liver were enzymatically determined with Cholesterol E-Test kits (Wako Pure Chemical Industries, Ltd).

\section{RNA extraction and quantitative real-time PCR}

Total RNA was extracted from the liver samples of the fishes in Experiment 1 by using RNeasy Lipid Tissue Mini-Kits (Qiagen K.K., Tokyo, Japan) and was transcribed into cDNA by using a High Capacity RNA-to-cDNA (Applied Biosystems, CA, USA). Quantitative real-time PCR was performed on an ABI PRISM platform (Applied Biosystems); analysis of cDNA samples employed the TaqMan Fast Universal PCR Master Mix. The TaqMan assays explored the expression levels of the following genes: eef1a1l1 (eukaryotic translation elongation factor 1 alpha 1, like 1; Dr03432748_m1); hmgcra (3-hydroxy-3-methylglutaryl-coenzyme A reductase A; Dr03428716_m1); $m t p$ (microsomal triglyceride transfer protein; Dr03133293_m1); and cyp7a1a (polypeptide 1a of subfamily A of family 7 of cytochrome P450; Dr03177268_s1). Each baseline and threshold level was manually set in line with the manufacturer's instructions. Relative mRNA expression levels were determined by using the expression level of eefla1l1 as an internal standard.

\section{Statistical analysis}

All data are shown as means \pm SE (standard error). The significance of observed differences was evaluated via analysis of variance followed by application of Fisher's partial least-squares difference multiple comparison. A difference was considered to be significant if the comparative $P$-value was $<0.05$. Statistical calculations were performed with the aid of Stat-View for Windows (version 5.0; SAS Institute Inc., NC, USA).

\section{Results}

Food intake and body weight of adult zebrafish

Food intake in Experiment 1 was estimated by daily observation. During the experimental period, all fish ate up each diet completely (20 mg of food/day per fish). Body weight increased significantly $(P<0.01)$ in each group over the experimental period, and the among-group differences were not significant (Figure 1).

\section{Plasma and liver cholesterol levels in adult zebrafish}

We examined plasma and liver cholesterol levels at the end of Experiment 1. The plasma cholesterol level was significantly higher $(P<0.01)$ in fish fed the highcholesterol diet $(722.3 \pm 75.5 \mathrm{mg} / \mathrm{dl})$ than in fish fed the control diet $(225.0 \pm 17.9 \mathrm{mg} / \mathrm{dl})$, but this rise was significantly suppressed $(P<0.01)$ in fish fed the CPP diet $(416.0 \pm 41.7 \mathrm{mg} / \mathrm{dl})$ (Figure 2A). The liver cholesterol level was significantly higher $(P<0.01)$ in fish fed the highcholesterol diet $(2.68 \pm 0.13 \mu \mathrm{g} / \mathrm{mg}$ liver weight $)$ than in fish fed the control diet $(2.10 \pm 0.11 \mu \mathrm{g} / \mathrm{mg}$ liver weight). This rise was significantly suppressed $(P<0.05)$ in fish fed the $\mathrm{CPP} \operatorname{diet}(2.32 \pm 0.15 \mu \mathrm{g} / \mathrm{mg}$ liver weight) (Figure $2 \mathrm{~B})$.

\section{Expression of mRNAs in the liver of adult zebrafish}

In an effort to define the mechanism by which CPPs exerted their anti-hypercholesterolemic effects, we measured the mRNA expression levels of genes associated with cholesterol metabolism in the liver. The levels of mRNA transcribed from hmgcra and $m t p$ were significantly lower $(P<0.01)$, and that from cyp7a1a significantly higher $(P<0.05)$, in fish fed the CPP diet than in fish fed the highcholesterol diet (Figure 3A, B, and C).

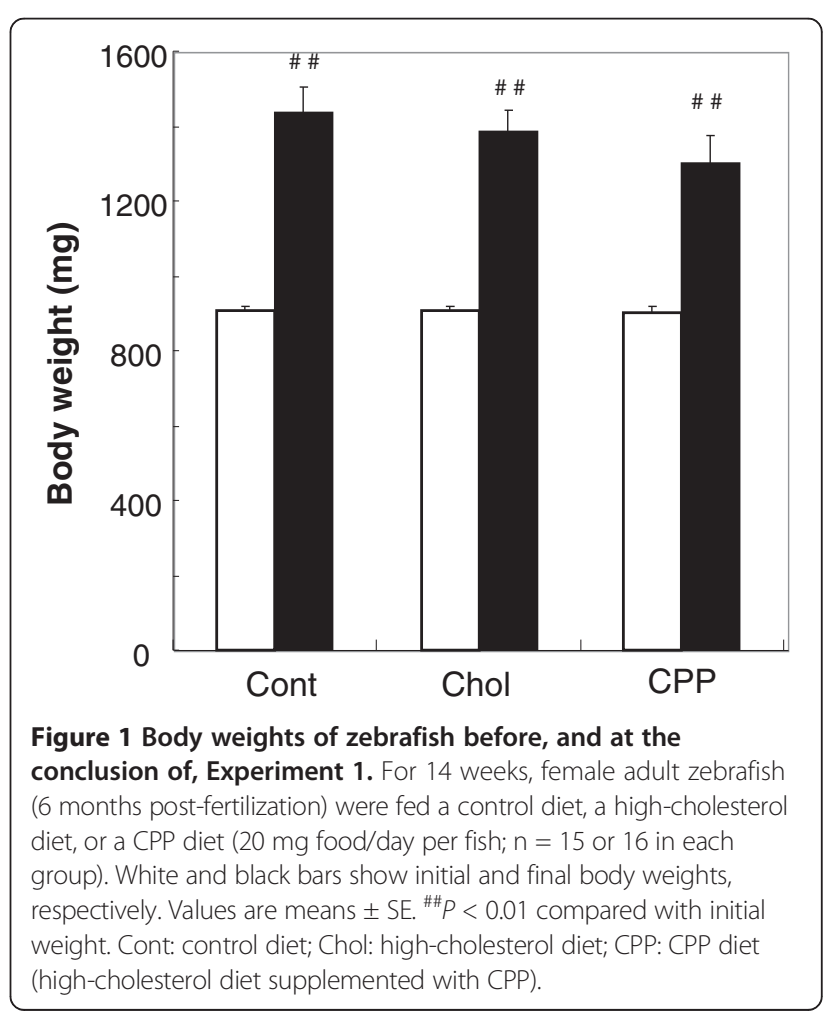



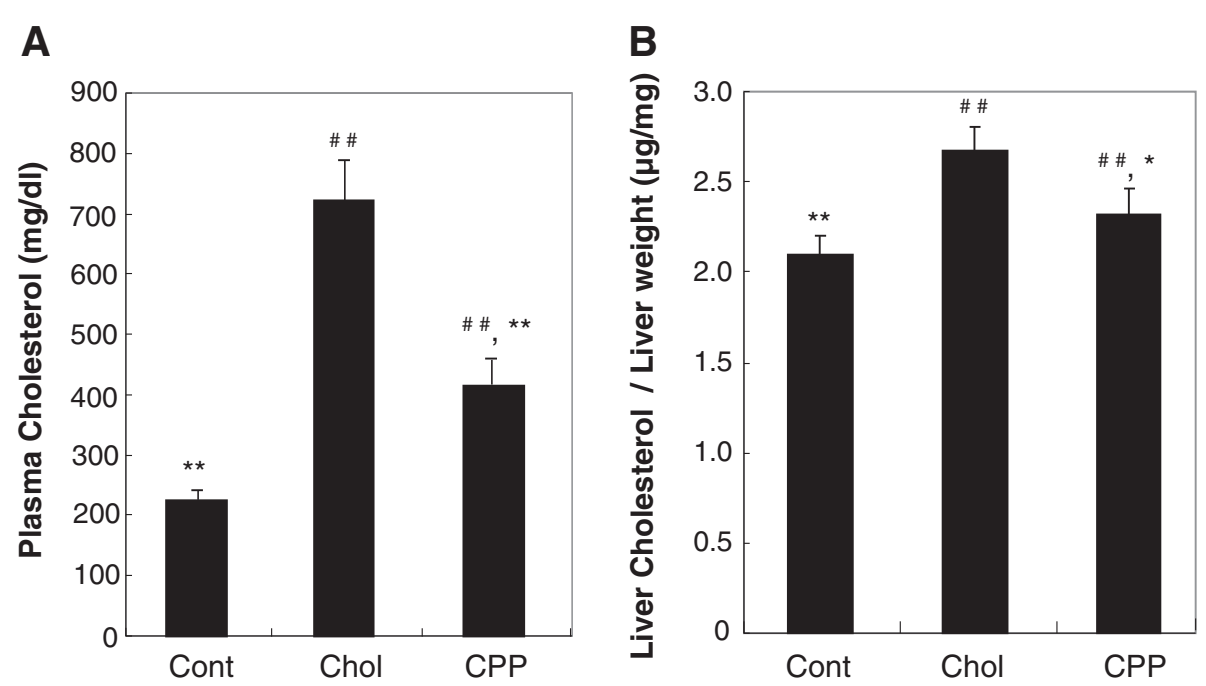

Figure 2 Effects of CPPs on plasma and liver cholesterol levels. Plasma (A) and liver (B) cholesterol levels are shown in fish fed a control diet, a high-cholesterol diet, or a CPP diet $\left(n=15\right.$ or 16 in each group). Values are means $\pm S E .{ }^{\# \#} P<0.01$ vs. fish fed a control diet; ${ }^{*} P<0.05$; ${ }^{* *} P<0.01$ vs. fish fed a high-cholesterol diet.

\section{Vascular cholesterol accumulation in larval zebrafish}

We explored the effect of CPP dietary supplementation on the extent of vascular cholesterol accumulation in the caudal arteries of optically transparent zebrafish larvae. Optical and fluorescence images are shown (Figure 4A and B, respectively). Cholesterol tagged with a fluorescent marker accumulated in the caudal artery (arrows in Figure 4B). Figure $4 \mathrm{C}$ shows fluorescence images from the trunk to the tail (including the caudal artery) of fish from each dietary group. The caudal artery fluorescence level of cholesterol tagged with a fluorescent marker was significantly greater $(P<0.01)$ in fish fed the high-cholesterol diet than in fish fed the control diet, but this increase was tempered significantly $(P<0.01)$ when the high-cholesterol diet was supplemented with CPPs (Figure 4D).

\section{Discussion}

We explored whether supplementation of a high-cholesterol diet with CPPs would mitigate the hypocholesterolemic effect of the former diet in zebrafish. Addition of CPPs to the high-cholesterol diet suppressed the increase in plasma cholesterol levels induced by the unsupplemented diet. Cho et al. [11] and Wan et al. [12] reported that addition of 5-CQA, a major component of CPPs, to high-fat or high-cholesterol diets suppressed the increases in plasma cholesterol levels normally seen when such

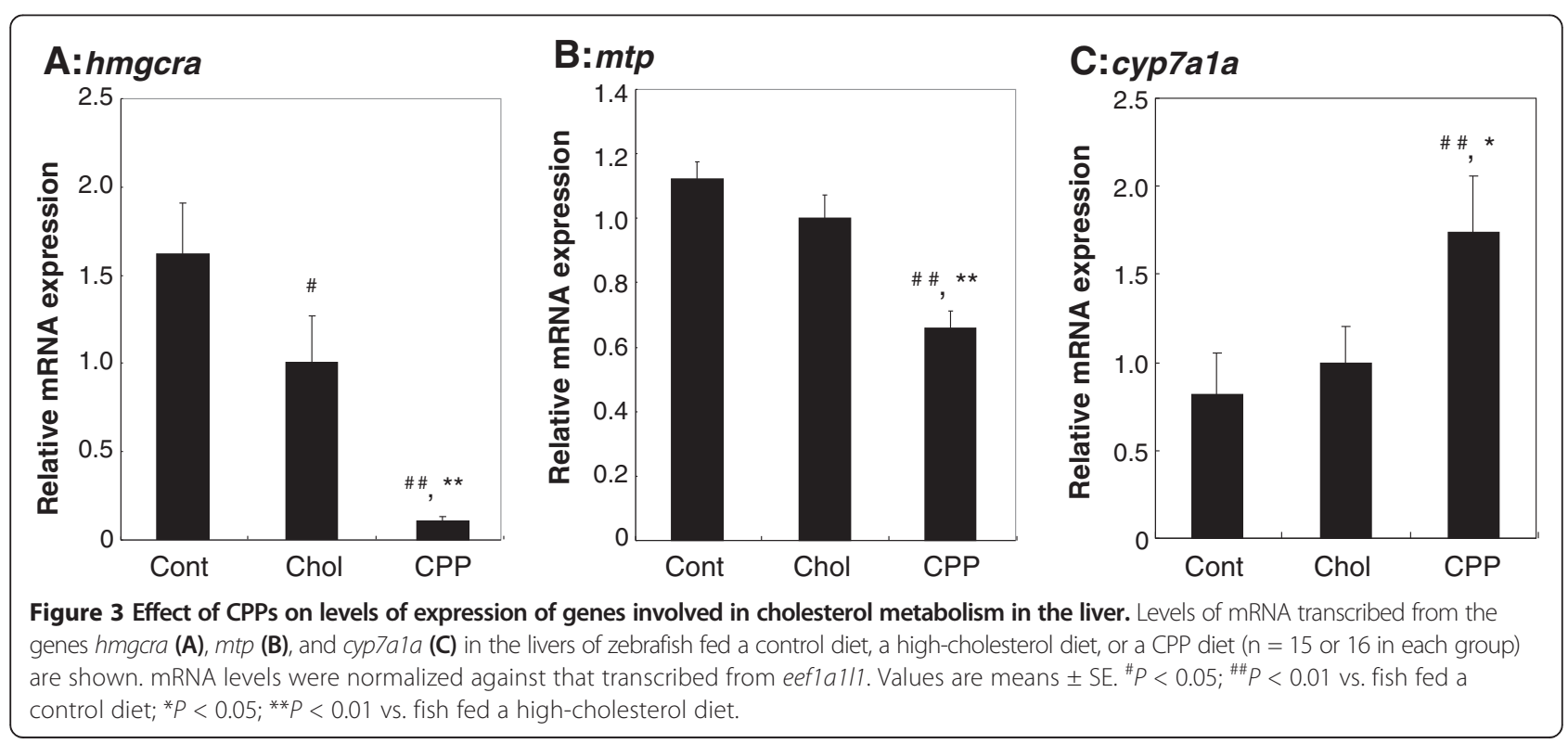



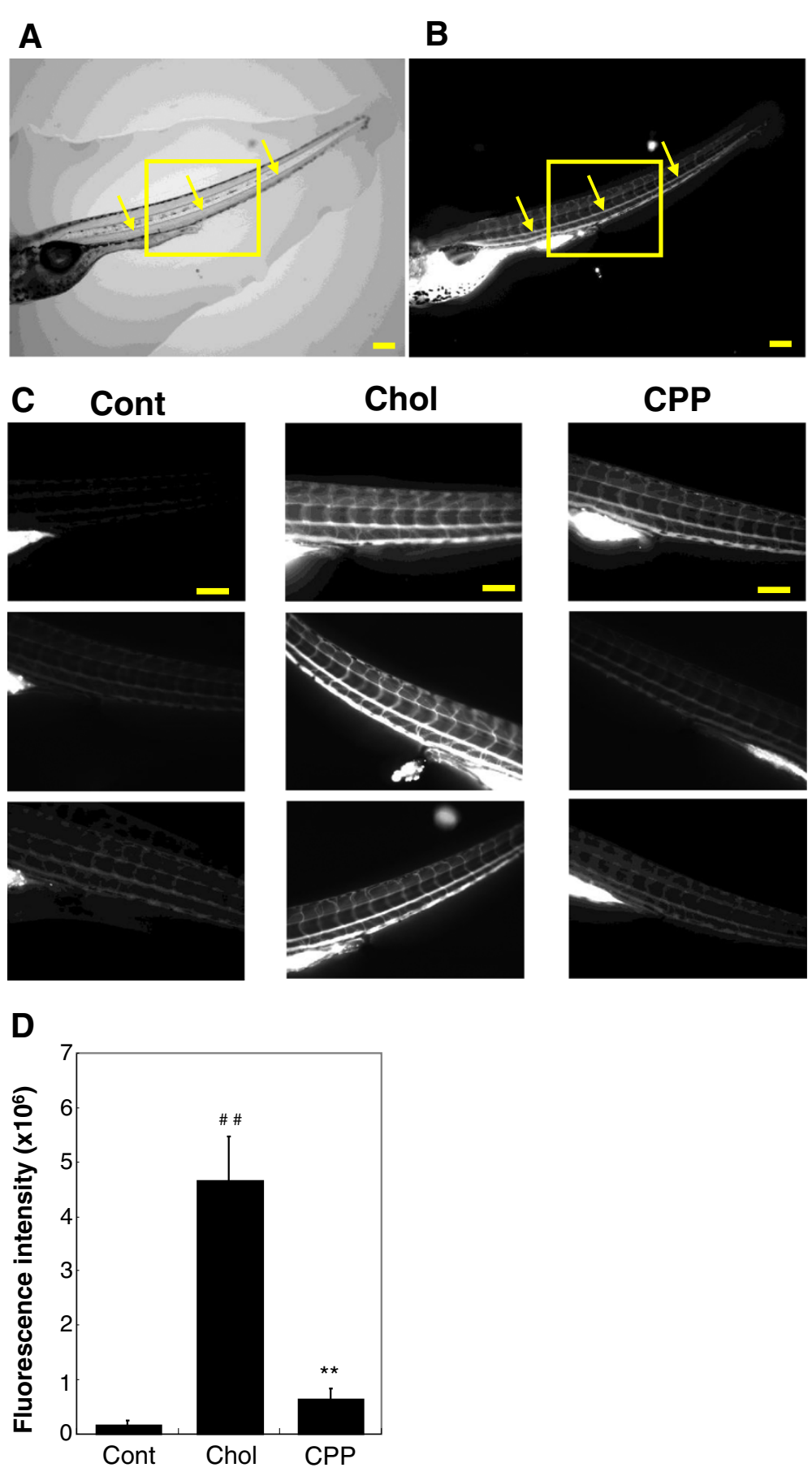

Figure 4 Effect of CPPs on vascular cholesterol accumulation in larval zebrafish. Larval zebrafish at 5 days post-fertilization were fed a control diet, a high-cholesterol diet, or a CPP diet; all diets additionally contained $0.002 \%(\mathrm{w} / \mathrm{w})$ of the fluorescent cholesteryl ester BODIPY 576/589 C11. Diets were administered over 10 days. A, B: Optical (A) and fluorescent (B) images taken of zebrafish larvae at the end of the experiment. Arrows in these images identify the caudal artery. Scale bars: $200 \mu \mathrm{m}$. C: Fluorescence images of zebrafish larvae from the trunk to the tail, including the caudal artery (outlined by rectangles in Figure 4A and B), taken after 10 days of consumption of a diet supplemented with fluorescent cholesterol. Scale bars: $200 \mu \mathrm{m}$. D: Fluorescence intensities of the caudal arteries of fish from the three dietary groups $(n=10)$. Values are means $\pm S E .{ }^{\# \#} P<0.01$ vs. fish fed a control diet; ${ }^{* *} P<0.01$ vs. fish fed a high-cholesterol diet. 
unsupplemented diets were fed to rodents. In our study, 5-CQA constituted $31.9 \%(\mathrm{w} / \mathrm{w})$ of the added CPPs; 5-CQA was thus the principal polyphenol present. Our results suggest that dietary CPPs lower plasma cholesterol levels partly because CPPs have high levels of 5-CQA. In addition, we found that CPP supplementation suppressed the increase in hepatic cholesterol level triggered by ingestion of a high-cholesterol diet. Murase et al. [9] also found that CPPs suppressed the accumulation of hepatic cholesterol in mice fed a high-fat diet. Together, the data suggest that CPPs exert a hypocholesterolemic effect by influencing cholesterol metabolism in the liver. CPPs ingested in food or drink (coffee) may be useful in prevention and management of hypercholesterolemia.

Hepatic mRNA expression analysis revealed that CPP supplementation decreased transcription of hmgcra and $m t p$ but elevated the transcriptional level of cyp7a1a. In the liver, the protein encoded by hmgcra is a rate-limiting enzyme in cholesterol biosynthesis and the target of statins, which are popular cholesterol-lowering drugs [22,23]. Thus, the observed decrease in expression of hmgcra may be one mechanism by which CPPs exert their hypocholesterolemic effects. On the other hand, mtp encodes an enzyme that is a lipid transfer protein required for the assembly and secretion of lipoproteins [24]. The protein encoded by cyp7a1a is rate-limiting enzyme for cholesterol catabolism; the enzyme converts cholesterol to bile acids [25]. Indeed, hypercholesterolemia induced by a high-cholesterol diet is not evident in liver-specific conditional Mttp knockout mice [26]. Transgenic mice overexpressing Cyp7a1 in the liver exhibit increased levels of hepatic cholesterol catabolism, a rise in the bile acid pool, and a decreased concentration of plasma cholesterol [27]. Therefore, we speculate that downregulation of cholesterol and lipoprotein synthesis, with concomitant upregulation of bile acid synthesis, underlies the hypocholesterolemic effects of CPP ingestion.

Cho et al. [11], cited above, showed that the hypocholesterolemic effects of 5-CQA could be attributed to a decrease in HMGCR enzymatic activity in the rodent liver. Such downregulation of activity, attributable to a fall in the level of mRNA transcription from $\mathrm{Hmgcr}$, has been suggested to be the fundamental mechanism whereby the dietary polyphenols epigallocatechin3-gallate, resveratrol, curcumin, and hesperetin exert hypocholesterolemic effects [28-33]. Although the structure-activity relationships of these polyphenols remain poorly understood, the accumulated data encourage us to speculate that polyphenolic compounds regulate hepatic $\mathrm{Hmgcr}$ expression and thus HMGCR levels. If so, the downregulation of hepatic hmgcra expression upon CPP dietary supplementation may be attributable not only to the action of 5-CQA, but also to the activities of other CPPs.
The caudal artery increase in accumulation of a fluorescent form of cholesterol in the fish fed a high-cholesterol diet was inhibited by concomitant ingestion of CPPs. Fang et al. [34] found that when larval zebrafish were fed a high-cholesterol diet for only 2 weeks the levels of certain oxidized cholesteryl esters, identical to those present in minimally oxidized LDL of humans and murine atherosclerotic lesions, rose by up to 70 times. In mammals, hypercholesterolemia increases the plasma levels of oxidized LDLs and lipid peroxides, in turn facilitating the adhesion of monocytes and lymphocytes to the vascular endothelium [35,36]. Notably, chlorogenic acid can inhibit the copper-induced oxidation of human LDL in vitro [37]. Chang et al. [38] found that chlorogenic acid suppressed the IL-1 $\beta$-induced expression of adhesion molecules and induced the production of reactive oxygen species. Therefore, the observed suppression of fluorescent cholesterol accumulation in the caudal artery upon dietary supplementation with CPPs may be attributable to the inhibition, by CPP components, of cholesterol or lipoprotein oxidation (or both) and to reduced expression of adhesion molecules of the vascular endothelium.

We showed here that CPPs exert hypocholesterolemic effects and inhibit cholesterol accumulation in blood vessels. However, the effects of coffee ingestion on hypercholesterolemia and cardiovascular disease remain controversial $[39,40]$. Although coffee is rich in CPPs, coffee contains several other potentially bioactive compounds, including caffeine, vitamin B3, magnesium, potassium, fibrous materials, and hydroxyhydroquinone [40,41]. This last compound, which is a generator of reactive oxygen species, compromises chlorogenic acid-induced improvements in blood pressure and endothelial function [42]. Further study is needed to define the coffee compounds exerting beneficial or detrimental effects on cholesterol metabolism and the vascular endothelial system.

\section{Conclusions}

We showed here that CPP ingestion by hypercholesterolemic zebrafish suppressed cholesterol accumulation in the plasma, liver, and vascular system via downregulation of cholesterol and lipoprotein synthesis and upregulation of the synthesis of bile acids. Our results suggest that CPPs can prevent the development of hypercholesterolemia in humans and that CPP ingestion may help in the management of this condition.

\section{Abbreviations}

CPPs: Coffee polyphenols; CQA: Caffeoylquinic acid; diCQA: Dicaffeoylquinic acid; FQA: Feruloylquinic acid; LDL: Low-density lipoprotein; Cyp7a1: Gene encoding cytochrome P450 polypeptide 1a of subfamily A of family 7 (Mus musculus); cyp7a1a: Gene encoding cytochrome P450 polypeptide 1a of subfamily A of family 7(Danio rerio); eefia1/1: Gene encoding eukaryotic translation elongation factor 1 alpha 1-like 1 (Danio rerio); hmgcra: Gene encoding 3-hydroxy-3-methylglutaryl-coenzyme A reductase A (Danio rerio); Hmgcr: Gene encoding 3-hydroxy-3-methylglutaryl-coenzyme A reductase 
(Mus musculus); mtp: Gene encoding microsomal triglyceride transfer protein (Danio rerio); Mttp: Gene encoding microsomal triglyceride transfer protein (Mus musculus); HMGCR: 3-hydroxy-3-methylglutaryl-coenzyme A reductase (Mus musculus).

\section{Competing interests}

No author has any competing interest to declare.

\section{Authors' contributions}

SM conducted all the animal studies. TH1 performed RNA extractions and quantitative real-time PCR analysis. SM drafted the manuscript with the help of the other authors. All authors were involved in conception of the study and participated in study design and coordination. All authors have read and approved the final manuscript.

\section{Authors' information}

Shinichi Meguro, PhD, is a senior principal research scientist in R\&D - Biological Science Research at Kao Corporation. Dr. Meguro's research focus is lipid metabolism, nutrition, and health science.

Takahiro Hasumura is a research scientist in R\&D - Biological Science Research at Kao Corporation. Mr. Hasumura's research focus is health science. Tadashi Hase DVM is a Vice-President with responsibility for R\&D - Biological Science Research at Kao Corporation. Dr. Hase's research focus is lipid metabolism, nutrition, and health science.

\section{Acknowledgments}

The authors thank Ms. Mari Tsutsumi for maintaining the zebrafish colony.

Received: 19 May 2013 Accepted: 26 September 2013 Published: 3 October 2013

\section{References}

1. van der Wulp MY, Verkade HJ, Groen AK: Regulation of cholesterol homeostasis. Mol Cell Endocrinol 2013, 368(1-2):1-16.

2. Gotto AM Jr, Moon JE: Management of cardiovascular risk: the importance of meeting lipid targets. Am J Cardiol 2012, 110:3A-14A.

3. Mannu GS, Zaman MJ, Gupta A, Rehman HU, Myint PK: Evidence of lifestyle modification in the management of hypercholesterolemia. Curr Cardiol Rev 2013, 9(1):2-14.

4. Johnston KL, Clifford MN, Morgan LM: Coffee acutely modifies gastrointestinal hormone secretion and glucose tolerance in humans: glycemic effects of chlorogenic acid and caffeine. Am J Clin Nutr 2003, 78(4):728-33.

5. Suzuki A, Yamamoto N, Jokura H, Yamamoto M, Fujii A, Tokimitsu I, Saito I: Chlorogenic acid attenuates hypertension and improves endothelial function in spontaneously hypertensive rats. J Hypertens 2006, 24(6):1065-73.

6. Suzuki A, Kagawa D, Ochiai R, Tokimitsu I, Saito I: Green coffee bean extract and its metabolites have a hypotensive effect in spontaneously hypertensive rats. Hypertens Res 2002, 25(1):99-107.

7. Yamaguchi T, Chikama A, Mori K, Watanabe T, Shioya Y, Katsuragi Y, Tokimitsu I: Hydroxyhydroquinone-free coffee: a double-blind, randomized controlled dose-response study of blood pressure. Nutr Metab Cardiovasc Dis 2008, 18(6):408-14.

8. Ochiai R, Jokura H, Suzuki A, Tokimitsu I, Ohishi M, Komai N, Rakugi H, Ogihara T: Green coffee bean extract improves human vasoreactivity. Hypertens Res 2004, 27(10):731-7.

9. Murase T, Misawa K, Minegishi Y, Aoki M, Ominami H, Suzuki Y, Shibuya Y, Hase T: Coffee polyphenols suppress diet-induced body fat accumulation by downregulating SREBP-1c and related molecules in C57BL/6J mice. Am J Physiol Endocrinol Metab 2011, 300(1):E122-33.

10. Murase T, Yokoi Y, Misawa K, Ominami H, Suzuki Y, Shibuya Y, Hase T: Coffee polyphenols modulate whole-body substrate oxidation and suppress postprandial hyperglycaemia, hyperinsulinaemia and hyperlipidaemia. Br J Nutr 2012, 107(12):1757-65.

11. Cho AS, Jeon SM, Kim MJ, Yeo J, Seo KI, Choi MS, Lee MK: Chlorogenic acid exhibits anti-obesity property and improves lipid metabolism in high-fat diet-induced-obese mice. Food Chem Toxicol 2010, 48(3):937-43.

12. Wan CW, Wong CN, Pin WK, Wong MH, Kwok CY, Chan RY, Yu PH, Chan SW: Chlorogenic acid exhibits cholesterol lowering and fatty liver attenuating properties by up-regulating the gene expression of ppar-a in hypercholesterolemic rats induced with a high-cholesterol diet. Phytother Res 2013, 27(4):545-551.
13. Rodriguez de Sotillo DVR, Hadley M: Chlorogenic acid modifies plasma and liver concentrations of: cholesterol, triacylglycerol, and minerals in (fa/fa) Zucker rats. J Nutr Biochem 2002, 13(12):717-726.

14. Karthikesan K, Pari L, Menon VP: Antihyperlipidemic effect of chlorogenic acid and tetrahydrocurcumin in rats subjected to diabetogenic agents. Chem Biol Interact 2010, 188(3):643-50.

15. Lieschke GJ, Currie PD: Animal models of human disease: zebrafish swim into view. Nat Rev Genet 2007, 8(5):353-67.

16. Anderson JL, Carten JD, Farber SA: Zebrafish lipid metabolism: from mediating early patterning to the metabolism of dietary fat and cholesterol. Methods Cell Biol 2011, 101:111-4.

17. Hölttä-Vuori M, Salo VT, Nyberg L, Brackmann C, Enejder A, Panula P, Ikonen E: Zebrafish: gaining popularity in lipid research. Biochem J 2010, 429(2):235-42.

18. Stoletov K, Fang L, Choi SH, Hartvigsen K, Hansen LF, Hall C, Pattison J, Juliano J, Miller ER, Almazan F, Crosier P, Witztum JL, Klemke RL, Miller YI: Vascular lipid accumulation, lipoprotein oxidation, and macrophage lipid uptake in hypercholesterolemic zebrafish. Circ Res 2009, 104(8):952-60.

19. Westerfield M, Streisinger G: THE ZEBRAFISH BOOK Edition 5 A guide for the laboratory use of zebrafish Danio* (Brachydanio) rerio. University of Oregon Press; 2007.

20. Guide for the Care and Use of Laboratory Animals Eight edition: The National Academies Press: 2010.

21. Folch J, Lees M, Sloane Stanley GH: A simple method for the isolation and purification of total lipids from animal tissues. J Biol Chem 1957, 226(1):497-509.

22. Lennernäs H, Fager G: Pharmacodynamics and pharmacokinetics of the HMG-CoA reductase inhibitors. Similarities and differences. Clin Pharmacokinet 1997, 32(5):402-425.

23. Grundy SM: Cholesterol-lowering drugs as cardioprotective agents. Am J Cardiol 1992, 70(21):27|-321.

24. Hussain MM, Bakillah A: New approaches to target microsomal triglyceride transfer protein. Curr Opin Lipidol 2008, 19(6):572-8.

25. Chiang JY: Bile acids: regulation of synthesis. J Lipid Res 2009, 50(10):1955-66.

26. Chang BH, Liao W, Li L, Nakamuta M, Mack D, Chan L: Liver-specific inactivation of the abetalipoproteinemia gene completely abrogates very low density lipoprotein/low density lipoprotein production in a viable conditional knockout mouse. J Biol Chem 1999, 274(10):6051-5.

27. Li T, Owsley E, Matozel M, Hsu P, Novak CM, Chiang JY: Transgenic expression of cholesterol 7alpha-hydroxylase in the liver prevents high-fat diet-induced obesity and insulin resistance in mice. Hepatology 2010, 52(2):678-90.

28. Cuccioloni M, Mozzicafreddo M, Spina M, Tran CN, Falconi M, Eleuteri AM, Angeletti M: Epigallocatechin-3-gallate potently inhibits the in vitro activity of hydroxy-3-methyl-glutaryl-CoA reductase. J Lipid Res 2011, 52(5):897-907.

29. Hirsova P, Kolouchova G, Dolezelova E, Cermanova J, Hyspler R, Kadova Z, Micuda S: Epigallocatechin gallate enhances biliary cholesterol secretion in healthy rats and lowers plasma and liver cholesterol in ethinylestradiol-treated rats. Eur J Pharmacol 2012, 691(1-3):38-45.

30. Cho IJ, Ahn JY, Kim S, Choi MS, Ha TY: Resveratrol attenuates the expression of HMG-CoA reductase mRNA in hamsters. Biochem Biophys Res Commun 2008, 367(1):190-4.

31. Shin SK, Ha TY, McGregor RA, Choi MS: Long-term curcumin administration protects against atherosclerosis via hepatic regulation of lipoprotein cholesterol metabolism. Mol Nutr Food Res 2011, 55(12):1829-40.

32. Yiu WF, Kwan PL, Wong CY, Kam TS, Chiu SM, Chan SW, Chan R: Attenuation of fatty liver and prevention of hypercholesterolemia by extract of Curcuma longa through regulating the expression of CYP7A1, LDL-receptor, HO-1, and HMG-CoA reductase. J Food Sci 2011, 76(3):H80-9.

33. Kim HK, Jeong TS, Lee MK, Park YB, Choi MS: Lipid-lowering efficacy of hesperetin metabolites in high-cholesterol fed rats. Clin Chim Acta 2003, 327(1-2):129-37.

34. Fang L, Harkewicz R, Hartvigsen K, Wiesner P, Choi SH, Almazan F, Pattison J, Deer E, Sayaphupha T, Dennis EA, Witztum JL, Tsimikas S, Miller YI: Oxidized cholesteryl esters and phospholipids in zebrafish larvae fed a high cholesterol diet: macrophage binding and activation. J Biol Chem 2010, 285(42):32343-51.

35. Owens AP 3rd, Passam FH, Antoniak S, Marshall SM, McDaniel AL, Rudel L, Williams JC, Hubbard BK, Dutton JA, Wang J, Tobias PS, Curtiss LK, Daugherty A, Kirchhofer D, Luyendyk JP, Moriarty PM, Nagarajan S, Furie BC, Furie B, Johns DG, Temel RE, Mackman N: Monocyte tissue factor-dependent activation of coagulation in hypercholesterolemic mice and monkeys is inhibited by simvastatin. J Clin Invest 2012, 122(2):558-68. 
36. Kita T, Kume N, Minami M, Hayashida K, Murayama T, Sano H, Moriwaki H, Kataoka H, Nishi E, Horiuchi H, Arai H, Yokode M: Role of oxidized LDL in atherosclerosis. Ann N Y Acad Sci 2001, 947:199-205.

37. Gordon MH, Wishart K: Effects of chlorogenic acid and bovine serum albumin on the oxidative stability of low density lipoproteins in vitro. J Agric Food Chem 2010, 58(9):5828-33.

38. Chang WC, Chen CH, Lee MF, Chang T, Yu YM: Chlorogenic acid attenuates adhesion molecules upregulation in IL-1 beta-treated endothelial cells. Eur J Nutr 2010, 49(5):267-75.

39. Bøhn SK, Ward NC, Hodgson JM, Croft KD: Effects of tea and coffee on cardiovascular disease risk. Food Funct 2012, 3(6):575-91.

40. Di Castelnuovo A, di Giuseppe R, lacoviello L, de Gaetano G: Consumption of cocoa, tea and coffee and risk of cardiovascular disease. Eur J Intern Med 2012, 23(1):15-25

41. Hiramoto K, Li X, Makimoto M, Kato T, Kikugawa K: Identification of hydroxyhydroquinone in coffee as a generator of reactive oxygen species that break DNA single strands. Mutat Res 1998, 419(1-3):43-51.

42. Suzuki A, Fujii A, Jokura H, Tokimitsu I, Hase T, Saito I:

Hydroxyhydroquinone interferes with the chlorogenic acid-induced restoration of endothelial function in spontaneously hypertensive rats. Am J Hypertens 2008, 21(1):23-7.

doi:10.1186/1743-7075-10-61

Cite this article as: Meguro et al.: Coffee polyphenols exert hypocholesterolemic effects in zebrafish fed a high-cholesterol diet. Nutrition \& Metabolism 2013 10:61.

\section{Submit your next manuscript to BioMed Central and take full advantage of:}

- Convenient online submission

- Thorough peer review

- No space constraints or color figure charges

- Immediate publication on acceptance

- Inclusion in PubMed, CAS, Scopus and Google Scholar

- Research which is freely available for redistribution 